\title{
Acute ischemic infarct in the middle cerebral artery territory following a Russell's viper bite
}

\author{
Sanjeev K. Narang, Srikanth Paleti, M. A. Azeez Asad, Tarannum Samina \\ Department of General Medicine, Mamata General Hospital, Mamata Medical College, Khammam, Andhra Pradesh, India
}

\author{
Address for correspondence: \\ Dr. Sanjeev K. Narang \\ 10-2-107, SVPD, Mamillagudem, \\ Khammam, Andhra Pradesh, India. \\ E-mail: narang_sanjeev@yahoo. \\ com
}

DOI: $10.4103 / 0028-3886.55594$

\begin{abstract}
Ischemic stroke following snake bite is rare. We report an 18-year male who developed right hemiplegia with expressive aphasia following a Russell's viper bite. T2-weighted magnetic resonance imaging revealed infarct in the left middle cerebral artery territory. The possible mechanisms for cerebral infarction in this scenario include disseminated intravascular coagulation, toxin induced vasculitis and endothelial damage.
\end{abstract}

Key words: Cerebral infarct, Russell's viper, snake bite

\section{Introduction}

In India more than 20,00,000 snake bites are reported annually, of which 35,000 to 50,000 people die. ${ }^{[1]}$ Russell's viper, Vipera russelli siamensis, is the leading cause of fatal snake bite in India. ${ }^{[2]}$ The clinical characteristics include local cellulitis, renal failure, and hemorrhagic manifestations including pituitary and intracranial hemorrhage. In this report we present an unusual complication, cerebral infarction following Russell's viper bite.

\section{Case Report}

An 18-year-old previously healthy male was admitted for a snake bite on his left foot which he sustained in his paddy fields. The killed snake was identified as Russell's viper. He complained of severe pain. Local examination showed two deep fang marks, erythema and edema. Neurological examination was essentially normal on admission. He was immediately treated with $30 \mathrm{ml}$ loading dose of equine polyvalent antisnake venom (ASV-ASIA, Bharat Serum and Vaccines Ltd.) after a test dose, followed by continuous intravenous administration of $30 \mathrm{ml}$ every 6 hours by syringe pump until clotting time normalized. A further dose of $30 \mathrm{ml}$ over 24 hours was given to prevent recurrence of abnormal coagulation profile. ${ }^{[3]}$ An injection of adsorbed tetanus toxoid was also given. Laboratory investigations: Mild leukocytosis, microscopic hematuria, prolonged bleeding time (5.00 $\mathrm{min})$ and clotting time (12:00 $\mathrm{min})$, prolonged prothrombin time (>3 min; control $16 \mathrm{sec}$ ) and activated plasma thromboplastin time ( $>180 \mathrm{sec}$; control $36 \mathrm{sec}$ ). Serum fibrinogen was $0.2 \mathrm{~g} / \mathrm{dL}$ and fibrin degradation products were negative. Clotting time was corrected in about 24 hours. Prothrombin time and activated plasma thromboplastin time were normalized by the fourth day.

On the second day of the bite, patient developed right side weakness and speech disturbances. Neurological examination revealed right hemiplegia and expressive aphasia. Brain computed tomography (CT) scan and magnetic resonance imaging (MRI) showed acute ischemic infarct in the left middle cerebral artery territory [Figure 1]. Magnetic resonance angiography of the cerebral circulation revealed no abnormalities. Colour Doppler study showed no arterial or venous thrombosis in the lower limbs. Work-up for other stroke risk profiles electrocardiogram (ECG), lipid profile including lipoprotein (a), serum homocystiene, proteins C, S, and antithrombin III, carotid Doppler and 2D echocardiography were normal.

Patient showed improvement in the motor power and speech at the time of discharge on the tenth day. At 14-months follow-up he had good functional recovery and is able to carry activities of daily living with little difficulty, modified Rankin score 1. 


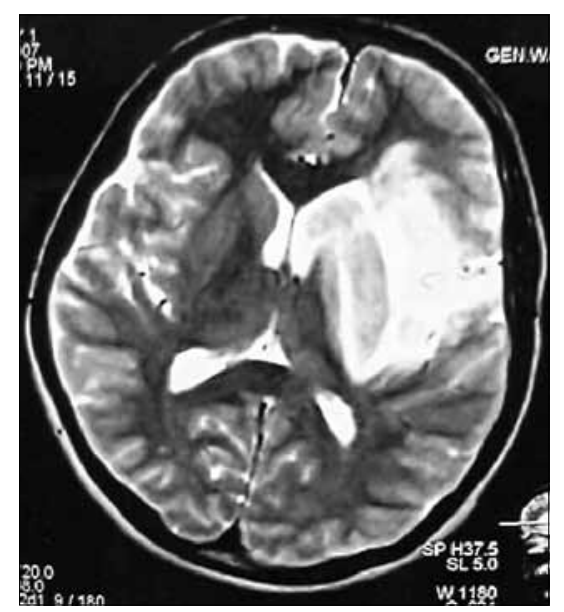

Figure 1: Magnetic resonance imaging of the brain showing acute ischemic infarct in the left MCA territory

\section{Discussion}

Cerebral complications, particularly ischemic complications, after snake bite are rare. Very few cases of cerebral infarction resulting from a viper bite have been reported. ${ }^{[4-6]}$ In a study of 309 patients with snake bite, Mosquera, et al. reported cerebrovascular complications in 8 patients $(2.6 \%), 7$ hemorrhagic strokes and 1 ischemic stroke. ${ }^{[4]}$ Bashir and Jinkins reported a patient in whom envenomation with Russell's viper resulted in hemiplegia and aphasia, consistent with a middle cerebral artery infarction. ${ }^{[5]}$ Murthy, et al. reported a case of cerebral infarction and diffuse encephalopathy following a viper bite. ${ }^{[6]}$

Viper snake venom is a complex toxin with rich components dominantly affecting hemostatic mechanisms. ${ }^{[7]}$ In large doses, it can cause massive intravascular coagulation leading to small and even large vessel occlusions resulting in cerebral infarction. ${ }^{[8]}$ Toxic vasculitis caused by certain viperine species may result in thrombosis. ${ }^{[6]}$ Bashir and Jinkins suggested direct action of the venom on vascular endothelial cells. ${ }^{[5]}$ Hemorrhagins, the complement mediated, toxic components of Viperidae snake venom may result in severe vascular spasm, endothelial damage, and increased vascular permeability, ${ }^{[5]}$ all of which may contribute to vascular occlusion. ${ }^{[6]}$ Hypercoagulation due to procoagulants in the venom, such as arginine, esterase, and hydrolase ${ }^{[5,6]}$ and hyperviscosity caused by hypovolemia and hypoperfusion secondary to hypotension may also contribute to vessel occlusion. The cerebral infarction may be totally unrelated and may be the manifestation of an inherent deficiency of protein C, protein S, and antithrombin III. ${ }^{[9]}$ Our patient was young and had no vascular risk factor and MR angiography was normal. We feel in our patient the cerebral infarction was the result of toxic vasculitis or toxin induced vascular spasm and endothelial damage.

Better outcomes have been reported with immediate ASV treatment. In the study by Thomas et al., of the 33 patients with envenomation by Bothrops lanceolatus who had not received ASV or received ASV after 8 hours of envenomation, $14 \%$ developed thrombotic complications and 4 of the 14 patients who had not received ASV died. Of the 70 patients who received ASV within 6 hours of envenomation, none thrombotic complications. ${ }^{[10]}$ Our patient, despite treatment with ASV within 1 hour of envenomation developed delayed cerebral infarction on the second day. Our case also illustrates that one should work up for possible cerebral infarction in a victim of viper envenomation and focal deficit.

\section{References}

1. David AW. Guidelines for the clinical management of snake-bites in the south-east Asia region. World Health Organization, Regional Office for South East Asia, New Delhi: 2005. p. 1-67.

2. Warrell DA. Snake venoms in science and clinical medicine. 1. Russell's viper: Biology, venom and treatment of bites. Trans R Soc Trop Med Hyg 1989;83:732-40.

3. Srimannarayana J, Dutta TK, Sahai A, Badrinath S. Rational Use of Anti-snake Venom (ASV): Trial of Various Regimens in Hemotoxic Snake Envenomation. J Assoc Physicians India 2004;52:788-93.

4. Mosquera A, Idrovo LA, Tafur A, Del Brutto OH. Stroke following Bothrops spp. snakebite. Neurology 2003;60:1577-80.

5. Bashir R, Jinkins J. Cerebral infarction in a young female following snake bite. Stroke 1985;16:328-30.

6. Murthy JM, Kishore LT, Naidu KS. Cerebral infarction after envenomation by viper. J Comput Assist Tomogr 1997;21:35-7.

7. Marsh NA. Snake venom affecting haemostatic mechanism - A consideration of their mechanism, practical applications and biological significance. Blood Coagul Fibrinolysis 1994;5:399-410.

8. Schwartzman RJ, Hill JB. Neurologic complications of disseminated intravascular coagulation. Neurology 1982;32:791-7.

9. Kumar V., Cortan RS., Robbins SL. Basic pathology. $5^{\text {th }}$ ed. Bangalore: Prism books PVT; 1992. p. 69-70.

10. Thomas L, Tyburn B, Ketterlé J, Biao T, Mehdaoui H, Moravie V, et al. Prognostic significance of clinical grading of patients envenomed by Bothrops lanceolatus in Martinique. Trans R Soc Trop Med Hyg 1998;92:542-5.

Accepted on 26-02-09

Source of Support: Nil, Conflict of Interest: None declared. 